\title{
Influence of physical activity on periodontal health in patients with type 2 diabetes mellitus. A blinded, randomized, controlled trial
}

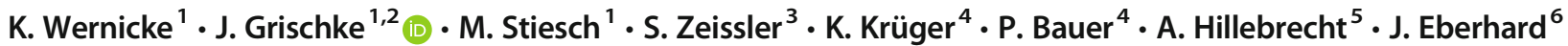

Received: 28 December 2020 / Accepted: 23 March 2021 / Published online: 1 April 2021

(C) The Author(s) 2021

\begin{abstract}
Objectives The aim was to investigate the effect of physical activity on periodontal health and $\mathrm{HbA1c}$ levels in patients with type 2 diabetes mellitus (T2DM) over a period of 6 months.

Materials and methods Thirty-seven patients with non-insulin-dependent T2DM were included in the study. The intervention group $(n=20)$ performed physical activity over a period of 6 months. The control group $(n=17)$ did not receive any intervention. Baseline and final examinations included dental parameters and concentrations of glycosylated hemoglobin (HbAlc) and highsensitivity C-reactive protein (hsCRP).

Results Physical activity showed a positive effect on periodontal health. Both the BOP $(p=0.005)$ and the severity of periodontitis $(p=0.001)$ were significantly reduced in the intervention group compared to the control group. Furthermore, HbA1c levels were reduced $(p=0.010)$ significantly in the intervention group while hsCRP levels significantly increased in the control group $(p=0.04)$.

Conclusions Within the limitations of this randomized, controlled trial, physical activity over a period of 6 months is a healthpromoting measure for patients with $\mathrm{T} 2 \mathrm{DM}$ and improves both periodontal health and $\mathrm{HbA} 1 \mathrm{c}$ concentrations.
\end{abstract}

Keywords Diabetes $\cdot$ Periodontitis $\cdot$ Physical activity $\cdot \mathrm{HbAlc} \cdot$ Periodontal health

\section{Introduction}

Diabetes mellitus is one of the most common chronic metabolic diseases and results from an absolute insulin deficiency,

K. Wernicke and Jasmin Grischke contributed equally to this work.

Clinical relevance Physical activity may contribute to periodontal health in addition to positive effects on systemic health in patients with T2DM.

J. Grischke

Grischke.Jasmin@mh-hannover.de

1 Hannover Medical School, Hanover, Germany

2 Department of Prosthetic Dentistry and Biomedical Materials Science, Hannover Medical School, Carl-Neuberg-Str.1, 30625 Hannover, Germany

3 Sportpark Zwickau, Zwickau, Germany

4 Justus-Liebig-Universität Gießen, Gießen, Germany

5 Volkswagen Nutzfahrzeuge, Hanover, Germany

6 The University of Sydney School of Dentistry and the Charles Perkins Centre, Faculty of Health and Medicine, The University of Sydney, Sydney, Australia insulin resistance, or defective insulin production. Type 2 diabetes mellitus (T2DM) is the most common chronic disease in the Western world. Already in the 1970s, studies found that T2DM patients were more likely to develop periodontal diseases than healthy people [1].

Diabetes has been identified as an important risk factor for periodontitis. The risk of developing periodontitis and periimplantitis is significantly increased in patients with diabetes compared to healthy control groups [2-5]. This phenomenon was described in various studies and a causal connection between T2DM and periodontitis was postulated [6-8]. In a clinical study, a significant reduction in blood glucose (HbAlc values) was found after non-surgical periodontal therapy in patients with moderate to advanced chronic periodontitis $[9,10]$. However, the exact causal mechanism has not yet been conclusively investigated. Possible links between diabetes and chronic periodontitis may include local and systemic inflammatory responses, the presence of advanced glycation end products (AGEs), oxidative stress, or mitochondrial dysfunction $[11,12]$. In addition, pathogenic bacteria and their by-products induce the synthesis of acute phase proteins, cytokines, and oxidative stress molecules in the liver, which subsequently reduce insulin sensitivity [7]. 
Studies including lifestyle and health interventions have shown that they have a positive health effect on patients with T2DM. For example, physical activity has been shown to improve the metabolic condition of T2DM patients, reduce $\mathrm{HbA} 1 \mathrm{c}$ levels and cardiovascular mortality, and improve the quality of life, lipid levels, and blood pressure [13, 14]. Periodontal health has also been significantly improved by physical activity in clinical trials $[15,16]$. Other studies found significantly lower plaque indices, gingival indices, and less clinical attachment loss in physically active subjects compared to less active subjects [17]. At the molecular level, reactive oxygen species (ROS) may play a role as a link between physical activity, T2DM, and periodontitis. In clinical studies, systemic ROS levels were reduced by physical activity, while chronic periodontitis had the opposite effect $[18,19]$. It was also shown that high systemic ROS concentrations are associated with decreased glycemic control [20].

To test the hypothesis that physical activity is a healthpromoting measure with significant positive effects on periodontal health and HbA1c concentrations, this examinerblinded, controlled clinical trial was conceived in subjects with non-insulin-dependent T2DM over a period of 6 months.

\section{Material and methods}

\section{Study population}

Participants $>18$ years old with non-insulin-dependent type 2 diabetes mellitus were recruited by information sessions in a gymnastic hall and advertisements at regional doctors in a medium-sized town in Germany (Zwickau, Saxony, Germany). The study aimed for the inclusion of a representative sample with respect to sex, age, and ethnicity. Informed oral and written consent was obtained from each participant.

\section{Inclusion and exclusion criteria}

This randomized clinical trial has been carried out in accordance with The Code of Ethics of the World Medical Association (Declaration of Helsinki) and was approved by the local Ethics Committee of the Justus-Liebig University Giessen, Germany (reference number 2011-0006). The study was performed by the Department of Sports Medicine of the University of Giessen, Germany, in collaboration with Hannover Medical School, Germany. The trial was registered at ClinicalTrials.gov (NCT 01377558).

T2DM was defined according to the WHO as described in [21]. Inclusion criteria were non-insulin-dependent T2DM and the willingness to participate in a baseline and follow-up dental examination. Exclusion criteria were unstable coronary artery disease, any serious medical condition that prevented adherence to the study protocol, or the ability to exercise safely, advanced retinopathy, and current insulin therapy. Furthermore, patients with preexisting physical activity of $\geq$ $60 \mathrm{~min}$ per week were not eligible.

\section{Exercise program}

We conducted a 26-week, single center, randomized, controlled trial with a parallel group design. Previously inactive persons with type 2 diabetes were randomly assigned to 1 of 4 groups: aerobic exercise, resistance training, combined aerobic and resistance training, or a control group that reverted to pre-study exercise levels.

Altogether, 126 patients with type 2 diabetes were willing to participate. They were randomly assigned and matched 1:1 to the four study groups. After exclusion of 16 patients as screening failure, 110 patients remained. Thirty patients were assigned to the aerobic exercise group (group AE), 27 to the resistance-training group (RE), 25 to the combined training group (CE), and 28 patients to the control group (CG).

All participants were provided with a 6-month membership at an exercise facility. The membership fees were covered by the study funding to remove economic barriers to participation. Individual exercise supervision was provided with a fitness coach twice a week throughout the study. Attendance was verified through direct observation, exercise logs, and individual smart card controllers. Participants with $>10 \%$ missed trainings were excluded.

The exercise was carried out twice a week for 6 months. Each exercise unit was preceded by a 10-min warm up period. The training duration and intensity gradually progressed after four and 13 weeks, respectively. The intervention groups exercised according to the study protocol of the assigned group. All participants in the intervention group took regularly part in the training.

The training method was based on published programs of the working group [22]. Intervention group 1 (strength endurance) completed a strength-endurance training after a general warm up and stretching in a group setting, followed by two passes of a strength-endurance circuit. The strength-endurance training consisted of eight machine-supported exercises that included all major muscle groups (leg abduction, leg adduction, back extension (lower back), dips, vertical row, vertical traction, leg press, abdominal crunch) and were performed for 1 min each. During the initial training session, a maximum force test with three attempts was performed. The best of the three tests was scored and used to define the exercise intensity at about $60 \%$ of the participant's maximum force for the first 2 weeks of training. For the subsequent weeks, the load was increased by $10 \%$ and again by $5 \%$ for the last 4 weeks. Intervention group 2 (endurance) completed a progressive endurance training 2 times a week. After a general warm up and stretching in a group setting a 30-min training on a treadmill (technogym run 500/technogym run 600) or a bicycle 
ergometer with or without backrest (lifefitness-lifecycle 9500 HR) was performed. Intervention group 3 (combination intervention) completed a training combining both programs: one time a week an endurance and one time a week a strength endurance training was performed. All participants of the intervention groups regularly participated in the training.

Participation, correct exercise performance and execution, and the compliance of the participants were supervised by professional trainers under supervision of a sport scientist with doctors' degree. All trainings took place in a certified sports center (certified by AG Diabetes, Sport und Bewegung der deutschen Gesellschaft für Geriatrie DGG).

All participants were advised not to change eating habits or to start diets during the study. Additionally, we took steps to minimize medication co-interventions by sending letters to participants' physicians to inform about the study. We asked to maintain any antihypertensive and lipid-lowering therapy during the 6 months exercise intervention period. Adaption of medical therapy was possible for medical reasons. Changes in glucose-lowering medication should be reported either by the participant or the physician to the study group. We did not initiate any changes in medication throughout the study and devices used during the study are depicted in Table 1. The control group did not receive a sports program or any other lifestyle intervention.

\section{Medical and dental records}

A qualified dentist interviewed the participants on pre-existing periodontal diseases and habits including smoking and frequencies of dental check-ups. Body mass index (BMI) and weight in kilograms were recorded at baseline and after 6 months. Furthermore, any history of periodontitis was recorded.

\section{Assessment of periodontal conditions}

For each patient, periodontal probing depth (PPD), bleeding on probing (BOP), and plaque scores were measured using a Florida probe system. PPD measurements were done at 6 sites per tooth (mesio-buccal, buccal, disto-buccal, mesio-oral, oral, and disto-oral) and BOP were recorded at 4 sites (mesio-buccal, disto-buccal, buccal, and oral). Furthermore, a periodontal anamnesis collected data on history of periodontitis. Periodontitis was classified according to Eke and Page et al. [23]. However, recently, a new international classification has been published. Severity of periodontal disease according to Eke and Page et al. (healthy, light, moderate, and severe) were denoted as (modified) stages $(1,2,3,4)$ according to Papapanou et al. (2018) without including a grading [24]. Full-mouth plaque index (API) was measured in \% [25].

\section{Blood sampling}

In addition to the dental examination, blood samples of each patient were collected in the morning after fasting and hsCRP and $\mathrm{HbA} 1 \mathrm{c}$ levels were measured in $\mathrm{mg} / \mathrm{L}$ or $\%$ at baseline and after 6 months. Blood analyses were performed in a certified clinical laboratory (Laboratory Community Saxony West, Zwickau, Germany) using the Blood Analyses Modular P800 (Roche-Institut). HbA1c levels were measured using an Adams A1c HA8180V Analyzer (Axon Lab AG, Reichenbach, Deutschland).

\section{Statistical analysis}

All data were electronically stored. The statistical analysis was carried out in Excel (Version 2102, Microsoft, USA). Due to the small number of patients in the sport intervention groups, all three sports interventions were pooled as the intervention group and compared with the control group irrespective of the training method. First, $t$ tests with equal variances were calculated to compare changes between baseline and the 6-month follow-up for participants' weight, BMI, HbA1c, hsCRP, BOP, and periodontal staging between the sport intervention and the control group. Second, a multivariate regression analysis was performed to identify predictors of for the clinical parameter BOP as a marker of acute periodontal inflammation. The significance level was set at $P<0.05$.
Table 1 An overview over the sport equipment that was accessible to the participants of the intervention group over a period of 6 months. The left table column summarizes the strength endurance equipment and the right column the cardio endurance devices

\begin{tabular}{lc}
\hline $\begin{array}{l}\text { Strength endurance devices (milon strength endurance } \\
\text { circle) }\end{array}$ & Cardio endurance devices \\
\hline Leg abduction & Treadmill (technogym run 500/technogym run \\
Leg adduction & 600 ) \\
Back extension (lower back) & Bicycle with backrest (lifefitness-lifecycle \\
& 9500 HR) \\
Dips-vertical row-vertical traction & Bicycle without backrest (lifefitness-lifecycle \\
Leg press & 9500 HR) \\
Abdominal crunch &
\end{tabular}




\section{Results}

A total number of 108 participants (women $n=65$, men $n=43$, age range 46 to 73 years) met the inclusion criteria and were randomized to the physical exercise or control groups. Thirtyone patients were excluded after the start of the study because patients did have no teeth $(n=5)$ or refused a dental examination $(n=26)$ leaving 77 at the baseline assessment. Thirtyseven participants were available for the final assessment with 20 participants of the physical activity group and 17 patients of the control group (Table 2). The mean $\mathrm{HbAlc}$ for all participants was $6.7 \%$ with a range between 5.6 and $9 \%$. A mean BMI of 32.2 with a range between 21.9 and 46.1 was calculated. The mean hsCRP concentration was $0.86 \mathrm{mg} / \mathrm{L}$ with a range between 0.1 and $6.1 \mathrm{mg} / \mathrm{L}$ at baseline.

\section{Univariate analysis}

No significant differences between the intervention and control group for changes between baseline and final assessment were found for weight $(P=0.103)$, BMI $(P=0.144)$, and plaque index $(P=0.06)$. Significant differences were found for HbAlc concentration $(P=0.011)$, hs CRP concentration $(P=0.040)$, BOP $(P=0.002)$, PPD $(P=<0.001)$, and periodontal staging $(P=<0.001)$. HbA1c levels, BOP, PPD, and periodontal staging significantly improved in the intervention compared to the control group. The hsCRP concentrations increased significantly in the control compared to the intervention group.

\section{Multivariate analysis}

In the multivariate analysis, all parameters were initially included in a statistical model (Tables 3 and 4) to identify independent risk factors for the variables BOP, periodontal staging, and $\mathrm{HbA} 1 \mathrm{c}$ concentrations.

A multivariate regression analyses with $\mathrm{HbAlc}$ as the outcome variable showed that $\mathrm{HbAlc}$ improved significantly in
Table 3 Multivariate regression analysis with (modified) periodontal staging after intervention as primary outcome. The significant results are printed in bold. The modified staging is significantly associated with the intervention group. History of periodontitis is an independent risk factor for more severe periodontitis. $C R P \mathrm{C}$-reactive protein, $B M I$ body mass index, $H b A l c$ glycated hemoglobin, $B O P$ bleeding on probing, staging classification into the periodontal severity according to Papapanou Sanz et al. (2018)

\begin{tabular}{|c|c|c|}
\hline & $t$ statistic & $p$ value \\
\hline CRP (final) & 0.315 & 0.755 \\
\hline $\mathrm{HbA} 1 \mathrm{c}$ in $\%$ (final) & 0.131 & 0.896 \\
\hline Intervention group & 3.757 & $<0.001$ \\
\hline Age & 0.874 & 0.389 \\
\hline Gender & 1.567 & 0.129 \\
\hline Smoking status & 0.012 & 0.990 \\
\hline BMI in $\mathrm{kg} / \mathrm{m}^{2}$ (final) & 1.921 & 0.066 \\
\hline Plaque index (final) & 1.085 & 0.287 \\
\hline BOP (final) & 1.675 & 0.106 \\
\hline Recall frequency & 0.262 & 0.795 \\
\hline History of periodontitis & 2.444 & 0.021 \\
\hline
\end{tabular}

study participants who participated in a sports intervention $(p$ $=0.037$ ) compared to control.

\section{Discussion}

The present study investigated whether a sports intervention over a period of 6 months is an oral and general healthpromoting measure in patients with T2DM. The results showed that a sports intervention of at least 6 months in patients with T2DM has a significant positive effect on periodontal health and HbA1c concentrations compared to a control group without sports intervention. The comparison of the sports intervention group with the control group showed a significant reduction in BOP, a significant improvement in the severity of periodontal disease, and significantly lower
Table 2 The results (mean values \pm standard deviations) of all participants who received dental examination at baseline and a final examination

\begin{tabular}{|c|c|c|c|c|}
\hline & \multicolumn{2}{|c|}{ Control $(N=17)$} & \multicolumn{2}{|c|}{ Intervention $(N=20)$} \\
\hline & Baseline & Final & Baseline & Final \\
\hline hbA1c (\%) & $6.64 \pm 0.57$ & $7.24 \pm 0.79$ & $6.71 \pm 0.79$ & $6.66 \pm 0.70$ \\
\hline hsCRP (mg/l) & $0.72 \pm 0.71$ & $2.35 \pm 3.62$ & $1.01 \pm 1.380$ & $1.03 \pm 1.51$ \\
\hline BMI $\left(\mathrm{kg} / \mathrm{m}^{2}\right)$ & $31.86 \pm 6.68$ & $33.11 \pm 6.92$ & $32.41 \pm 4.39$ & $31.34 \pm 4.61$ \\
\hline BOP (\#sites) & $1.35 \pm 1.88$ & $3.41 \pm 6.19$ & $14.5 \pm 19.84$ & $2.4 \pm 4.09$ \\
\hline Staging & $1,29 \pm 0.46$ & $1,53 \pm 0.50$ & $1.55 \pm 0.50$ & $1.1 \pm 0.3$ \\
\hline PPD (mm) & $1.41 \pm 0.50$ & $1.44 \pm 0.54$ & $2.27 \pm 0.60$ & $1.38 \pm 0.28$ \\
\hline Plaque (\%) & $21.64 \pm 21.16$ & $12.76 \pm 15.24$ & $26.90 \pm 27.68$ & $32.5 \pm 26.39$ \\
\hline Weight (kg) & $92.24 \pm 20.10$ & $96.03 \pm 19.64$ & $92.47 \pm 14.93$ & $89.33 \pm 15.74$ \\
\hline
\end{tabular}


Table 4 Multivariate regression with BOP after 6 months as primary outcome variable. The significant results are printed in bold. Lack of physical activity and the plaque index are independent risk factors for BOP. $C R P$ high sensitivity C-reactive protein, $B M I$ body mass index, $H b A l c$ glycated hemoglobin, $B O P$ bleeding on probing, staging classification into the periodontal severity according to Papapanou Sanz et al. (2018)

\begin{tabular}{lrr}
\hline CRP (final) & 0.507 & 0.616 \\
\hline HbA1c in \% (final) & 1.582 & 0.126 \\
Intervention group & 4.133 & $<\mathbf{0 . 0 0 1}$ \\
Age & 0.513 & 0.611 \\
Gender & 0.477 & 0.637 \\
Smoking status & 1.065 & 0.297 \\
BMI in kg/m ${ }^{2}$ (final) & 1.119 & 0.273 \\
Plaque index (final) & 5.323 & $<\mathbf{0 . 0 0 1}$ \\
History of periodontitis & 1.555 & 0.132 \\
Recall frequency & 1.353 & 0.187 \\
PPD (final) & 1.211 & 0.236 \\
\hline
\end{tabular}

$\mathrm{HbA} 1 \mathrm{c}$ levels in the intervention group at the final examination.

These results are consistent with the results of recent studies that have shown that physical activity is associated with a reduced prevalence of periodontitis and improved periodontal health of the population [26-28]. A study by Su-Jin Han et al. [27] showed a significant association between regular walking and a lower prevalence of periodontitis. Merchant et al. [28] confirmed these results. However, these studies have investigated the association between periodontitis and physical activity in a population without T2DM; to the knowledge of the authors, a study including patients at risk for T2DM has not been published yet.

The present study showed that physical activity was associated with improved periodontal health in patients with T2DM. However, a causal link between periodontitis, T2DM, and physical activity was not investigated. In the future, predictive models and individualized treatment options may be developed for patients with T2DM and periodontitis with the help of artificial intelligence and machine learning [29].

The significant reduction of BOP and the improvement of PA staging in the sports intervention groups may indicate that inflammatory processes in the oral cavity can be reduced by regular physical activity. However, periodontitis history and the plaque index were identified as independent predictors of BOP and the severity of periodontal disease as well.

Periodontitis and peri-implantitis are characterized by an inflammatory reaction, triggered by the host response to the pathologic oral microbiota with destruction of tissues $[3,30$, 31]. Health-promoting measures such as brushing teeth, flossing, healthy nutrition, and regular dental examinations are important to prevent or contain the development and progression of oral infectious disease $[15,32,33]$. In line with the results of the current study, other clinical studies have already shown that physical activity is considered a health-promoting measure and even leads to a reduction in the prevalence of periodontitis in the general population [15]. This reduction in prevalence could be related to the effects of physical activity on cytokine production and immune modulation [34-36]. Studies in patients with cardiovascular disease have shown that physical activity modulates multiple cytokines, in particular CRP, which is a by-product of the liver metabolism and plays an key role in the acute phase response. An elevated level of this inflammatory biomarker is associated with diabetes. Recent studies found an association between increased CRP levels in patients with periodontitis and other systemic conditions such as coronary heart disease, stroke, or diabetes mellitus [37, 38]. Not only CRP but also interleukin 1 beta (IL-1) is related to the inflammatory response of several disease $[36,39]$. Interestingly, regular training is an important prerequisite for maintaining cytokine levels and it has been shown that cytokine levels tend to return to their initial levels after 30 days without physical activity [40]. Remarkably, in a study by Qiu et al. (2014), the authors reported that regular exercise significantly lowers $\mathrm{HbA} 1 \mathrm{c}$ concentrations in T2DM [41] as well as the systolic blood pressure after a 6-month exercise program [42]; however, they did not investigate the effects on periodontal health.

In both groups, the BMI did not change significantly during the study. We found an increase of 1.2 in the control group and a decrease of 1.1 in the intervention group. This may be a trend; however, there was no significant correlation to the groups. Possible changes in muscle to fat ratio were not analyzed. Independent of the BMI, systematic inflammation and glycemic control improved with periodontal healing.

From 108 participants, only 77 met the inclusion criteria. Unfortunately, there was also a high number of dropouts during the course of the study. Only 37 participants were examined over the whole period. The retention rate of the study was extremely low for the dental examinations. We believe that this was due to the extra time of $30 \mathrm{~min}$ that was necessary for the dental examination. Furthermore, the dental examination was scheduled at the end of the medical examinations which contributed to a reduced compliance of the participants. Consequently, in future studies, this should be addressed beforehand by special incentives to increase motivation for dental examinations. A limitation of the study is that we did not investigate oral hygiene behaviors at baseline and follow-up. It may be possible that participants were particularly motivated and that the health-promoting measures, such as eating habits or tooth brushing habits, were changed and may in part explain the positive effects observed. 


\section{Conclusion}

In summary, the results of the present study showed that physical activity is an oral health-promoting measure in patients with T2DM and physical activity significantly reduces $\mathrm{HbA1c}$ concentrations. Still, the lasting effect on oral health and the possible causal link remains unclear. In future clinical trials, it would be interesting to compare the treatment success of periodontal treatment with and without accompanying sports intervention.

Author contribution All authors contributed to the study conception and design. Material preparation and data collection were performed by Katharina Wernicke. The first draft of the manuscript was written by Jasmin Grischke and Katharina Wernicke. All authors commented on previous versions of the manuscript. All authors read and approved the final manuscript.

Funding Open Access funding enabled and organized by Projekt DEAL. The work was supported by the authors' institutions.

\section{Declarations}

Ethics approval and consent to participate All procedures performed in this study were in accordance with the ethical standards of the institutional research committee of the Justus-Liebig University Giessen, Germany (reference number 2011-0006) and with the 1964 Helsinki declaration and its later amendments.

Informed consent was obtained from all individual participants included in the study.

Conflict of interest The authors declare no competing interests.

Open Access This article is licensed under a Creative Commons Attribution 4.0 International License, which permits use, sharing, adaptation, distribution and reproduction in any medium or format, as long as you give appropriate credit to the original author(s) and the source, provide a link to the Creative Commons licence, and indicate if changes were made. The images or other third party material in this article are included in the article's Creative Commons licence, unless indicated otherwise in a credit line to the material. If material is not included in the article's Creative Commons licence and your intended use is not permitted by statutory regulation or exceeds the permitted use, you will need to obtain permission directly from the copyright holder. To view a copy of this licence, visit http://creativecommons.org/licenses/by/4.0/.

\section{References}

1. Cohen DW, Friedman LA, Shapiro J, Kyle GC, Franklin S (1970) Diabetes mellitus and periodontal disease: two-year longitudinal observations part I. J Periodontol 41:709-712

2. Watanabe K (2011) Periodontitis in diabetics: is collaboration between physicians and dentists needed? Dis Mon 57:206-213

3. Dreyer H, Grischke J, Tiede C, Eberhard J, Schweitzer A, Toikkanen SE, Glöckner S, Krause G, Stiesch M (2018) Epidemiology and risk factors of peri-implantitis: a systematic review. J Periodontal Res 53:657-681. https://doi.org/10.1111/jre. 12562
4. Genco RJ, Borgnakke WS (2020) Diabetes as a potential risk for periodontitis: association studies. Periodontol (83):40-45

5. Preshaw PM, Bissett SM (2019) Periodontitis and diabetes. Br Dent J 227:577-584

6. Preshaw PM, Foster N, Taylor JJ (2007) Cross-susceptibility between periodontal disease and type 2 diabetes mellitus: an immunobiological perspective. Periodontol 45:138-157

7. Preshaw P, Alba A, Herrera D, Jepsen S, Konstantinidis A, Makrilakis K, Taylor R (2012) Periodontitis and diabetes: a twoway relationship. Diabetologia 55:21-31

8. Borgnakke WS (2019) IDF Diabetes Atlas: diabetes and oral health-a two-way relationship of clinical importance. Diabetes Res Clin Pract 157:107839

9. Yun F, Firkova EI, Jun-Qi L, Xun H (2007) Effect of non-surgical periodontal therapy on patients with type 2 diabetes mellitus. Folia Med (Plovdiv) 49:32-36

10. Navarro-Sanchez AB, Faria-Almeida R, Bascones-Martinez A (2007) Effect of non-surgical periodontal therapy on clinical and immunological response and glycaemic control in type 2 diabetic patients with moderate periodontitis. J Clin Periodontol 34:835843

11. Borgnakke WS, Ylöstalo PV, Taylor GW, Genco RJ (2013) Effect of periodontal disease on diabetes: systematic review of epidemiologic observational evidence. J Periodontol 84:S135-S152

12. Bullon P, Newman HN, Battino M (2014) Obesity, diabetes mellitus, atherosclerosis and chronic periodontitis: a shared pathology via oxidative stress and mitochondrial dysfunction? Periodontol 64:139-153

13. Pai L-W, Li T-C, Hwu Y-J, Chang S-C, Chen L-L, Chang P-Y (2016) The effectiveness of regular leisure-time physical activities on long-term glycemic control in people with type 2 diabetes: a systematic review and meta-analysis. Diabetes Res Clin Pract 113:77-85

14. Umpierre D, Ribeiro PA, Kramer CK, Leitao CB, Zucatti AT, Azevedo MJ, Gross JL, Ribeiro JP, Schaan BD (2011) Physical activity advice only or structured exercise training and association with $\mathrm{HbA} 1 \mathrm{c}$ levels in type 2 diabetes: a systematic review and meta-analysis. Jama 305:1790-1799

15. Al-Zahrani MS, Borawski EA, Bissada NF (2005) Increased physical activity reduces prevalence of periodontitis. J Dent 33:703-710

16. Eberhard J, Stiesch M, Kerling A, Bara C, Eulert C, HilfikerKleiner D, Hilfiker A, Budde E, Bauersachs J, Kück M (2014) Moderate and severe periodontitis are independent risk factors associated with low cardiorespiratory fitness in sedentary nonsmoking men aged between 45 and 65 years. J Clin Periodontol 41:31-37

17. Bawadi H, Khader Y, Haroun T, Al-Omari M, Tayyem R (2011) The association between periodontal disease, physical activity and healthy diet among adults in Jordan. J Periodontal Res 46:74-81

18. Dahiya P, Kamal R, Gupta R, Bhardwaj R, Chaudhary K, Kaur S (2013) Reactive oxygen species in periodontitis. J Indian Soc Periodontol 17:411

19. Joseph A-M and Hood DA (2014) Relationships between exercise, mitochondrial biogenesis and type 2 diabetes. Book title. Karger Publishers

20. Fatani SH, Babakr AT, NourEldin EM, Almarzouki AA (2016) Lipid peroxidation is associated with poor control of type-2 diabetes mellitus. Diabetes Metab Syndr Clin Res Rev 10:S64-S67

21. Lorenzo C, Okoloise M, Williams K, Stern MP, Haffner SM (2003) The metabolic syndrome as predictor of type 2 diabetes: the San Antonio heart study. Diabetes Care 26:3153-3159

22. Wasserfurth P, Palmowski J, Hahn A, Krüger K (2020) Reasons for and consequences of low energy availability in female and male athletes: social environment, adaptations, and prevention. Sports Med Open 6:44. https://doi.org/10.1186/s40798-020-00275-6 
23. Page RC, Eke PI (2007) Case definitions for use in populationbased surveillance of periodontitis. J Periodontol 78:1387-1399

24. Papapanou PN, Sanz M, Buduneli N, Dietrich T, Feres M, Fine DH, Flemmig TF, Garcia R, Giannobile WV, Graziani F (2018) Periodontitis: consensus report of workgroup 2 of the 2017 World Workshop on the Classification of Periodontal and Peri-Implant Diseases and Conditions. J Periodontol 89:S173-S182

25. Lange DE, Plagmann HC, Eenboom A, Promesberger A (1977) Clinical methods for the objective evaluation of oral hygiene. Dtsch Zahnarztl Z 32:44-47

26. Wernicke K, Zeissler S, Mooren FC, Frech T, Hellmann S, Stiesch M, Grischke J, Linnenweber S, Schmidt B, Menne J (2018) Probing depth is an independent risk factor for HbAlc levels in diabetic patients under physical training: a cross-sectional pilot-study. BMC Oral Health 18:46

27. Han S-J, Bae K-H, Lee H-J, Kim S-J, Cho H-J (2019) Association between regular walking and periodontitis according to socioeconomic status: a cross-sectional study. Sci Rep 9:1-7

28. Merchant AT, Pitiphat W, Rimm EB, Joshipura K (2003) Increased physical activity decreases periodontitis risk in men. Eur $\mathrm{J}$ Epidemiol 18:891-898

29. Grischke J, Johannsmeier L, Eich L, Griga L, Haddadin S (2020) Dentronics: towards robotics and artificial intelligence in dentistry. Dent Mater 36:765-778

30. Kabir L, Stiesch M, Grischke J (2020) The effect of keratinized mucosa on the severity of peri-implant mucositis differs between periodontally healthy subjects and the general population: a crosssectional study. Clin Oral Investig 25:1183-1193. https://doi.org/ 10.1007/s00784-020-03422-1

31. Foitzik M, Stumpp SN, Grischke J, Eberhard J, Stiesch M (2014) Evaluation of FTA® paper for storage of oral meta-genomic DNA. Biopreserv Biobank 12:337-342

32. Ferreira RO, Corrêa MG, Magno MB, Almeida APCPSC, Fagundes NCF, Rosing CK, Maia LC, Lima RR (2019) Physical activity reduces the prevalence of periodontal disease: systematic review and meta-analysis. Front Physiol 10:234

33. Bunk D, Eisenburger M, Häckl S, Eberhard J, Stiesch M, Grischke $\mathrm{J}$ (2020) The effect of adjuvant oral irrigation on self-administered oral care in the management of peri-implant mucositis: a randomized controlled clinical trial. Clin Oral Implants Res 31:946-958
34. Fernandes RA, Ritti-Dias RM, Balagopal PB, Conceição RD, Santos RD, Cucato GG, Bittencourt MS (2018) Self-initiated physical activity is associated with high sensitivity C-reactive protein: a longitudinal study in 5030 adults. Atherosclerosis 273:131-135

35. Malali E, Basar I, Emekli-Alturfan E, Elemek E, Oktay S, Ayan F, Emekli N, Noyan U (2010) Levels of C-reactive protein and protein $\mathrm{C}$ in periodontitis patients with and without cardiovascular disease. Pathophysiol Haemost Thromb 37:49-54

36. Sanders AE, Slade GD, Fitzsimmons TR, Bartold PM (2009) Physical activity, inflammatory biomarkers in gingival crevicular fluid and periodontitis. J Clin Periodontol 36:388-395

37. Beck JD, Moss KL, Morelli T, Offenbacher S (2018) Periodontal profile class is associated with prevalent diabetes, coronary heart disease, stroke, and systemic markers of C-reactive protein and interleukin-6. J Periodontol 89:157-165

38. Torrungruang K, Ongphiphadhanakul B, Jitpakdeebordin S, Sarujikumjornwatana S (2018) Mediation analysis of systemic inflammation on the association between periodontitis and glycaemic status. J Clin Periodontol 45:548-556

39. Hashioka S, Inoue K, Hayashida M, Wake R, Oh-Nishi A, Miyaoka $\mathrm{T}$ (2018) Implications of systemic inflammation and periodontitis for major depression. Front Neurosci 12:483

40. Maleki BH, Tartibian B, Mooren FC, FitzGerald LZ, Krüger K, Chehrazi M, Malandish A (2018) Low-to-moderate intensity aerobic exercise training modulates irritable bowel syndrome through antioxidative and inflammatory mechanisms in women: results of a randomized controlled trial. Cytokine 102:18-25

41. Qiu S, Cai X, Schumann U, Velders M, Sun Z, Steinacker JM (2014) Impact of walking on glycemic control and other cardiovascular risk factors in type 2 diabetes: a meta-analysis. PLoS One 9: e109767

42. Mandini S, Conconi F, Mori E, Myers J, Grazzi G, Mazzoni G (2018) Walking and hypertension: greater reductions in subjects with higher baseline systolic blood pressure following six months of guided walking. PeerJ 6:e5471

Publisher's note Springer Nature remains neutral with regard to jurisdictional claims in published maps and institutional affiliations. 